\title{
Enhanced stability of tetratic phase due to clustering
}

\author{
Yuri Martínez-Ratón* \\ Grupo Interdisciplinar de Sistemas Complejos (GISC), Departamento de Matemáticas, \\ Escuela Politécnica Superior, Universidad Carlos III de Madrid, \\ Avenida de la Universidad 30, 28911-Leganés, Madrid, Spain \\ Enrique Velascd $\rfloor^{\dagger}$ \\ Departamento de Física Teórica de la Materia Condensada and Instituto de Ciencia de Materiales Nicolás Cabrera, \\ Universidad Autónoma de Madrid, E-28049 Madrid, Spain
}

(Dated: September 24, 2008)

\begin{abstract}
We show that the relative stability of the nematic tetratic phase with respect to the usual uniaxial nematic phase can be greatly enhanced by clustering effects. Two-dimensional rectangles of aspect ratio $\kappa$ interacting via hard interactions are considered, and the stability of the two nematic phases (uniaxial and tetratic) is examined using an extended scaled-particle theory applied to a polydispersed fluid mixture of $n$ species. Here the $i$-th species is associated with clusters of $i$ rectangles, with clusters defined as stacks of rectangles containing approximately parallel rectangles, with frozen internal degrees of freedom. The theory assumes an exponential cluster size distribution (an assumption fully supported by Monte Carlo simulations and by a simple chemical-reaction model), with fixed value of the second moment. The corresponding area distribution presents a shoulder, and sometimes even a well-defined peak, at cluster sizes approximately corresponding to square shape (i.e. $i \simeq \kappa$ ), meaning that square clusters have a dominant contribution to the free energy of the hard-rectangle fluid. The theory predicts an enhanced region of stability of the tetratic phase with respect to the standard scaled-particle theory, much closer to simulation and to experimental results, demonstrating the importance of clustering in this fluid.
\end{abstract}

PACS numbers: $61.30 . \mathrm{Cz}, 61.30 . \mathrm{Hn}, 61.20 . \mathrm{Gy}$

\section{INTRODUCTION}

The hard rectangle (HR) fluid constitutes a paradigmatic example of a two-dimensional fluid exhibiting surprisingly complex phase behavior: different phase symmetries, phase transitions with different order, and defect-mediated continuous transitions of the KosterlitzThouless type [1], all governed solely by entropy. This peculiar two-dimensional system has three equilibrium fluid phases: isotropic (I), where particle axes are randomly oriented, uniaxial nematic $\left(\mathrm{N}_{\mathrm{u}}\right)$, with particles preferentially aligned along a single nematic director, and tetratic nematic $\left(\mathrm{N}_{\mathrm{t}}\right)$, possessing two equivalent perpendicular nematic directors, with long particle axes oriented along one of two directors with equal probability.

In a pioneering study, Schlacken et al. [2] applied scaled-particle theory (SPT) on a fluid of HRs to demonstrate the stability of the $\mathrm{N}_{\mathrm{t}}$ phase, a phase which cannot be stabilised in a fluid of hard ellipses. The intersection between the two spinodals associated with the $\mathrm{I}-\mathrm{N}_{\mathrm{t}}$ and I-N $\mathrm{N}_{\mathrm{u}}$ transitions defines a limiting aspect ratio $\kappa=L / \sigma_{0}$ (with $L$ and $\sigma_{0}$ the length and width of the rectangles, respectively) for the stability of the $\mathrm{N}_{t}$ phase, which is located at $\kappa \simeq 2.62$. Thus, for lower values of aspect ratio, the isotropic fluid exhibits a continuous transition to the $\mathrm{N}_{\mathrm{t}}$ phase, whereas if $\kappa>2.62$ the I phase goes

\footnotetext{
*Electronic address: yuri@math.uc3m.es

†Electronic address: enrique.velasco@uam.es
}

directly to the uniaxial nematic phase $\mathrm{N}_{\mathrm{u}}$.

The study of Schlacken et al. was later supplemented by the calculation of the complete phase diagram [3], also within the context of SPT. It was found, in particular, that the $\mathrm{N}_{\mathrm{t}}$ phase undergoes a transition to the $\mathrm{N}_{\mathrm{u}}$ phase at high density, the nature of which changes from second to first order at a tricritical point. In addition, it was shown [3] that, at the level of a particular approximation for density-functional theory, the $\mathrm{N}_{\mathrm{t}}$ fluid is metastable with respect to a phase with (either partial or complete) spatial order; the theory was approximate in the sense that it included the exact functional form of two-body correlations, but only approximate higher-order correlations. On the other hand, Monte Carlo (MC) simulations conducted on hard squares [4] and on a HR system [5] with $\kappa=2$ indicated, as expected, that the fluid exhibits quasi-long-range tetratic order, and that the highdensity phase consists of an aperiodic crystalline tetratic phase exhibiting random tiling on a square lattice. MC simulation of a HR fluid confined in a slit pore [6] show the presence of weak tetratic correlations at the centre of the pore.

In the experimental front, recent results for colloidal discs forced to stand on edge by external potentials [1] (and hence interacting approximately as HRs) have also demonstrated that tetratic correlations play a vital role in this system. Also, experiments conducted on a monolayer of vibrated granular cylinders lying on a plate [7] have shown tetratic correlations for cylinders with aspect ratio as high as $\kappa=12.6$.

In Ref. [8] strong evidence, based on MC simulations, 
was presented for the thermodynamic stability of a $\mathrm{N}_{\mathrm{t}}$ fluid when $\kappa$ is at least as large as 7 . These simulations were supplemented by an extended SPT model that exactly incorporates the second and third virial coefficients while resumming the remainder of the virial series. The inclusion of the third virial coefficient increases the interval in $\kappa$ where the $\mathrm{N}_{\mathrm{t}}$ phase is stable, approaching the simulation result. Specifically, the $\mathrm{I}_{\mathrm{N}} \mathrm{N}_{\mathrm{t}}$ transition line moves to lower packing fractions, while the intersection point between the $\mathrm{I}-\mathrm{N}_{\mathrm{u}}$ and the $\mathrm{I}-\mathrm{N}_{\mathrm{t}}$ transitions shifts to $\kappa=3.23$. However, this value is still lower than the value indicated by simulations.

The properties of the HR fluid are to be contrasted with those of hard discorectangles. MC simulations of this system have been conducted [9], and the global phase diagram was computed. A careful inspection of particle configurations in the isotropic phase shows that there are peculiar equilibrium textures, with large clusters containing particles arranged side by side, exactly as in the HR fluid. These configurations are favoured by the particular shape of the particles and by the reduced dimensionality. However, in contrast with the HR fluid, neighbouring clusters do not exhibit strong tetratic correlations and, therefore, the formation of a tetratic nematic phase is discouraged in the hard discorectangle fluid.

In this article we address the problem of how the present theoretical understanding of the HR fluid can be improved by consideration of clustering effects. Our thesis is that these effects, very apparent in our own MC simulations but not addressed by the theories proposed up to now, are a key factor in the stabilisation of the $\mathrm{N}_{\mathrm{t}}$ phase. Inclusion of cluster formation is responsible for the enhancement of the region of $\mathrm{N}_{\mathrm{t}}$ stability in the phase diagram. In the model proposed, clustering is approximately taken care of by treating clusters as distinct species in a mixture of polydispersed rectangles. The functional form for the cluster size distribution is assumed to be exponential, an assumption supported by cluster statistical results based on MC simulations and by a simple chemical-reaction model (see Appendix), and is introduced in the model as an input. The thermodynamics of the polydispersed mixture is analysed using SPT. The results indicate that clustering (assimilated in the theory by means of a polydispersity parameter) stabilises the $\mathrm{N}_{\mathrm{t}}$ phase for values of aspect ratio much higher than $\kappa=2.62$ if the polydispersity is sufficiently high. Polydispersity parameters obtained from simulation give support to the model.

The article is organized as follows. In Section II we present numerical evidence that the size distribution in the HR fluid is an exponentially decaying function. Section III presents the main ideas of the theoretical model proposed. The conclusions are drawn in Section IV. Finally, details on the model and on the procedure of solution are relegated to Appendices A and B, while Appendix C contains a chemical-reaction model for monomer aggregation which also supports the assumption of an exponential cluster size distribution.

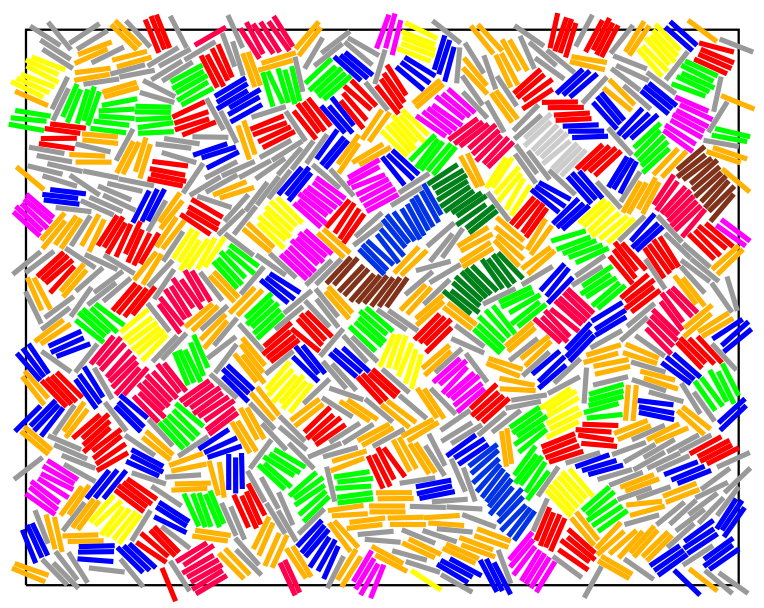

FIG. 1: (Colour online). Configuration of hard rectangles of aspect ratio $\kappa=7$ at packing fraction $\eta=0.655$, as obtained from MC simulation. Clusters, defined by the pair connectedness criterion explained in the text, have been coloured according to their size.

\section{MONTE CARLO SIMULATION OF CLUSTERING}

We started by applying standard isobaric (NPT) MC techniques on a two-dimensional fluid of hard rectangles, with aspect ratios $\kappa=3,5$ and 7 , using $N=1400$ rectangles. The transition from the I phase to the $\mathrm{N}_{\mathrm{t}}$ phase was identified approximately by inspection of the tetratic order parameter $q_{2}=\langle\cos 4 \phi\rangle$, where $\phi$ is the angle between the long axis of the particle and an axis fixed in space. Once the samples were equilibrated, cluster statistics was applied. The criterion for pair connectedness, i.e. for deciding when two neighbouring rectangles can be considered to be 'bonded', was based on the relative angle $\phi_{12}$ between the long axes of the particles and their relative centre-of-mass distance $\mathbf{r}_{12}$, by demanding that $\phi_{12}<\delta$ and $\left|\mathbf{r}_{12}\right|<\epsilon$. Typical values adopted were $\delta=10^{\circ}$ and $\epsilon=1.3 \sigma_{0}$, although the conclusions to be presented below do not seem to depend qualitatively on the exact values (provided they are not too large).

Fig. 1 presents a configuration of rectangles with $\kappa=7$ at a packing fraction $\eta=0.655$. This corresponds to a tetratic phase. Identified clusters have been coloured according to their size. One can see how these clusters look like large "super-rectangles" arranged along two perpendicular directions. Therefore, the tetratic structure is maintained not only for single rectangles, but also at the level of clusters ("polydispersed super-rectangles"). This hierarchical feature of the tetratic symmetry will give support to the theoretical model to be presented in the following section. In Fig. 2 a logarithmic histogram of the size distribution (averaged over configurations) is shown; in the figure, $x_{i}$ is defined as the fraction of clusters of size $i$ (see next section). The distribution looks exponential. All the curves in the figure pertain to a 


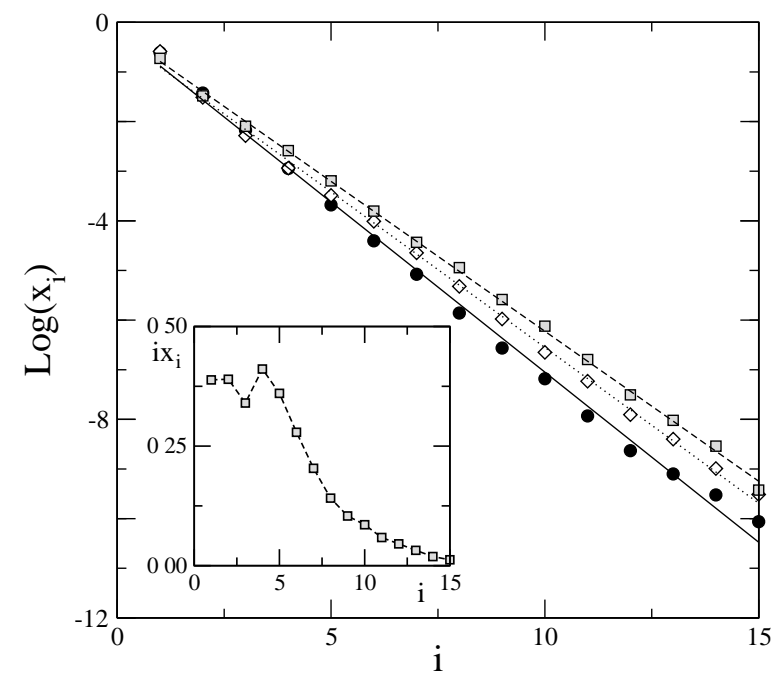

FIG. 2: Size distribution function $x_{i}$ as obtained from MC simulation, in logarithmic scale, for a fluid of HRs with different values of aspect ratio and packing fraction: filled circles, $\kappa=3$ and $\eta=0.635$; open squares, $\kappa=7$ and $\eta=0.600$; and grey squares, $\kappa=5$ and $\eta=0.657$. Straight lines are linear fits. Inset: area distribution function $i x_{i}$ for the case $\kappa=5$ and $\eta=0.692$.

tetratic phase, but the same behaviour is observed also in the isotropic phase (with the general trend that, for given $\kappa$, the slope decreases, i.e. the size distribution function becomes wider, hence the average cluster size, as density increases). In the size region corresponding to square clusters (i.e. clusters with aggregation number $i \sim \kappa)$ it is possible to see an incipient shoulder that grows as density is increased. This feature is more apparent in the area distribution function, $i x_{i}$ (giving the fraction of area occupied by clusters of a given size), which presents a shoulder and sometimes even a well-defined peak (inset of Fig. 2), indicating that square clusters are structurally very relevant and contribute very decisively to the thermodynamic properties of the fluid.

We end this section by discussing the orientational distribution functions. Let $h_{m}(\phi)$ be the monomer orientational distribution function, giving the probability of finding a given rectangle with its long axis forming an angle $\phi$ with respect to the director. Having defined clusters in the fluid, we may also define an orientational distribution function associated with clusters, $h_{c}(\phi)$, giving the probability of finding an average cluster (regardless of its size) oriented with angle $\phi$. In the simulations we compute $h_{c}(\phi)$ by averaging over all the identified clusters, and then over all MC configurations. Fig. 3 gives the functions $h_{m}(\phi)$ and $h_{c}(\phi)$ for a state with tetratic symmetry. It is interesting to note that these functions are almost identical (with the same symmetry and, consequently, with peaks with the same height), so that the structure of tetratic ordering is maintained from the monomer level to the cluster level, the functions $h_{m}(\phi)$ and $h_{c}(\phi)$ obeying a kind of "similarity" property. This

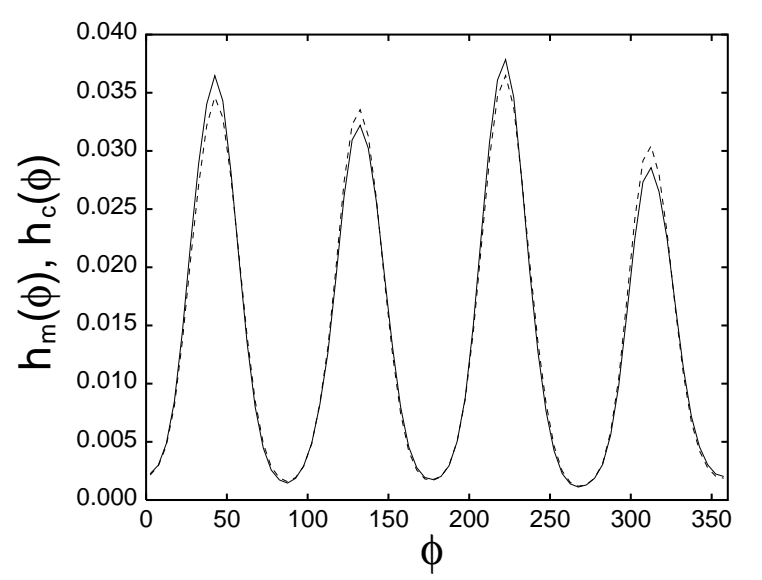

FIG. 3: Monomer $h_{m}(\phi)$ (solid line) and cluster $h_{c}(\phi)$ (dashed line) orientational distribution functions for the case $\kappa=7$ and $\eta=0.655$, as obtained from MC simulation.

property does not seem to be followed in the uniaxial nematic phase.

\section{THEORETICAL MODEL}

The model we propose accounts for clustering effects in an approximate way. The clear identification of clusters in the simulations, along with their approximate rectangular shape, leads to a simple model where clusters are regarded as single rectangular particles with no internal degrees of freedom. Therefore, we consider a $n-$ component mixture of two-dimensional hard rectangles of dimensions $L$ and $\sigma_{i}$, with $\sigma_{i}=i \sigma_{0}$ and $i$ the aggregation number. Each of these rectangles is assumed to be composed of $i$ monomers in perfect contact in a side-byside configuration. The total number of monomers, $N_{0}$, can be written as

$$
N_{0}=\sum_{i=1}^{n} i N_{i},
$$

where $N_{i}$ is the number of clusters containing $i$ monomers. Dividing by the volume $V$,

$$
\rho_{0}=\sum_{i=1}^{n} i \rho_{i},
$$

where $\rho_{0}=N_{0} / V$ is the total monomer density, and $\rho_{i}=N_{i} / V=x_{i} \rho$ is the density of clusters of size $i$, with $x_{i}=N_{i} / N$ their number fraction, while $N=\sum_{i} N_{i}$ is the total number of clusters and $\rho=N / V$ their density. The set $\left\{x_{i}\right\}, i=1, \cdots, n$, is a central quantity in our model, since it contains information about clustering tendencies. We will assume $x_{i}$ to be an exponential with $i$, as explained later. The total packing fraction of the 


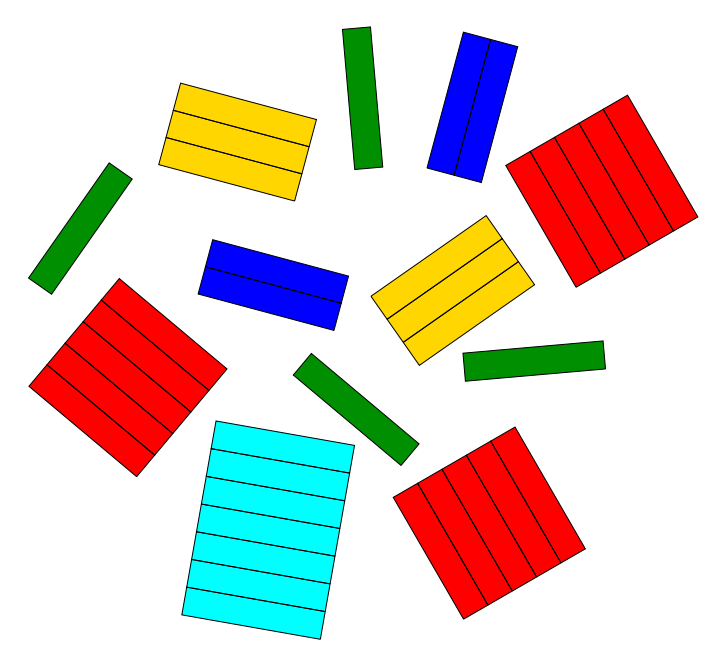

FIG. 4: (Colour online). Multicomponent isotropic mixture composed of different species, where species, depicted in different colours, correspond to clusters of a particular size. Clusters are defined as aggregates of rectangular monomers in a side-by-side configuration. In this instance the monomer aspect ratio is $\kappa=5$.

system is

$$
\eta=\sum_{i=1}^{n} \rho_{i} a_{i}
$$

where $a_{i}=i a_{0}\left(a_{0}=L \sigma_{0}\right)$ is the area of a cluster of size $i$. Using (2), we easily obtain $\eta=\rho_{0} a_{0}$.

Fig. 4 shows a schematic representation of an isotropic configuration of clusters of different sizes (with monomer aspect ratio $\kappa=5$ ). The original monomers that give rise to each cluster are indicated but note that, in our model, the identity of the monomers is lost, as monomers in the same cluster do not interact dynamically, always being in perfect side-by-side contact.

The free-energy of this multicomponent mixture of hard rectangles will be modelled by means of SPT applied to a fluid mixture of freely-rotating hard rectangles. The orientational properties of the mixtures will be characterised by orientational distribution functions $h_{i}(\phi)$ for each component $i$. The free-energy density functional $\Phi=\beta F / V$ is written as

$$
\begin{aligned}
\Phi\left[\left\{h_{i}\right\}\right] & =\rho\left\{\sum_{i=1}^{n} x_{i}\left[\ln \left(x_{i} \mathcal{V}_{i}\right)+\int_{0}^{\pi} d \phi h_{i}(\phi) \ln \left[h_{i}(\phi) \pi\right]\right]\right. \\
& \left.-1+\ln y+y S\left[\left\{h_{i}\right\}\right]\right\},
\end{aligned}
$$

where $\mathcal{V}_{i}$ is the thermal volume of $i$-sized clusters, and we defined $y=\rho a_{0} /(1-\eta)$. Due to the head-tail symmetry of the particles, the angle $\phi$ can be restricted to the interval $[0, \pi]$ and the functions $h_{i}(\phi)$ normalised accordingly.
The function $S\left[\left\{h_{i}\right\}\right]=\sum_{i, j} x_{i} x_{j} S_{i j}\left[\left\{h_{i}\right\}\right]$, with

$$
\begin{aligned}
S_{i j}\left[\left\{h_{i}\right\}\right] & =\frac{1}{2}\left(\kappa+i j \kappa^{-1}\right)\left\langle\left\langle\left|\sin \phi_{i j}\right|\right\rangle\right\rangle \\
& +\frac{1}{2}(i+j)\left\langle\left\langle\left|\cos \phi_{i j}\right|\right\rangle\right\rangle,
\end{aligned}
$$

is related to $A_{i j}$, the angle-averaged excluded area between clusters $i$ and $j$, as $S_{i j}=\left(A_{i j} / a_{0}-i-\right.$ $j) / 2$. The shorthand notation $\left\langle\left\langle f\left(\phi_{i j}\right)\right\rangle\right\rangle$ has been used for the double angular average of a generic function: $\left\langle\left\langle f\left(\phi_{i j}\right)\right\rangle\right\rangle=\int_{0}^{\pi} d \phi_{i} \int_{0}^{\pi} d \phi_{j} h_{i}\left(\phi_{i}\right) h_{j}\left(\phi_{j}\right) f\left(\phi_{i j}\right)$. Now a bifurcation analysis of (4) at the $\mathrm{I}_{-} \mathrm{N}_{u, t}$ transition (see Appendix) allows us to obtain the packing fractions of the I-N $\mathrm{N}_{u, t}$ spinodal lines as

$$
\eta^{*}=\left[1-\frac{4}{\pi} g_{k}\left(\frac{\kappa}{m_{0}^{(1)}}+\frac{m_{0}^{(2)}}{\kappa m_{0}^{(1)}}+2(-1)^{k}\right)\right]^{-1},
$$

with $k=1$ for the uniaxial and $k=2$ for the tetratic nematic, while $m_{0}^{(\alpha)}=\sum_{i} x_{i} i^{\alpha}(\alpha=1,2)$, are the first and second moments of the cluster size distribution function.

Based on the MC results, we adopt an exponential cluster size distribution:

$$
x_{i}=\frac{1-q}{1-q^{n}} q^{i-1}, \quad i=1, \cdots, n,
$$

with $q=e^{-\lambda}(\lambda>0)$. The prefactor in (7) ensures that the distribution is normalised, i.e. that $\sum_{i} x_{i}=1$. The first two moments can be derived analytically:

$$
\begin{aligned}
& m_{0}^{(1)}=\frac{1-[1+n(1-q)] q^{n}}{(1-q)\left(1-q^{n}\right)} \\
& m_{0}^{(2)}=\frac{1+q-\left[q+(1+n(1-q))^{2}\right] q^{n}}{(1-q)^{2}\left(1-q^{n}\right)} .
\end{aligned}
$$

Now we use (6) to obtain the maximum aspect ratio which can support a stable tetratic phase. This follows by imposing the condition $\eta_{0, t}^{*}=\eta_{0, u}^{*}$, i.e. by searching for the intersection point of the two spinodal lines $\mathrm{I}-\mathrm{N}_{\mathrm{u}}$ and $\mathrm{I}-\mathrm{N}_{\mathrm{t}}$ in the phase diagram $\eta-\kappa$. Solving for the corresponding value of $\kappa$, we obtain

$$
\kappa=\frac{1}{2}\left(3 m_{0}^{(1)} \pm \sqrt{9\left(m_{0}^{(1)}\right)^{2}-m_{0}^{(2)}}\right)
$$

In the specific case where the number of species goes to infinity, $n \rightarrow \infty$, we obtain from (8) $-(10)$

$$
\Delta=\kappa^{-1}\left[\frac{1}{2}\left(2 \kappa^{2}-3 \kappa-1 \pm \sqrt{\kappa^{2}+6 \kappa+1}\right)\right]^{1 / 2}
$$

where $\Delta=\sqrt{m_{0}^{(2)} /\left(m_{0}^{(1)}\right)^{2}-1}=\sqrt{q}$, a measure of polydispersity, is the relative mean square deviation. The two functions $\Delta(\kappa)$, corresponding to the two signs in (11), are plotted in Fig. 5 (note that one of the branches, 


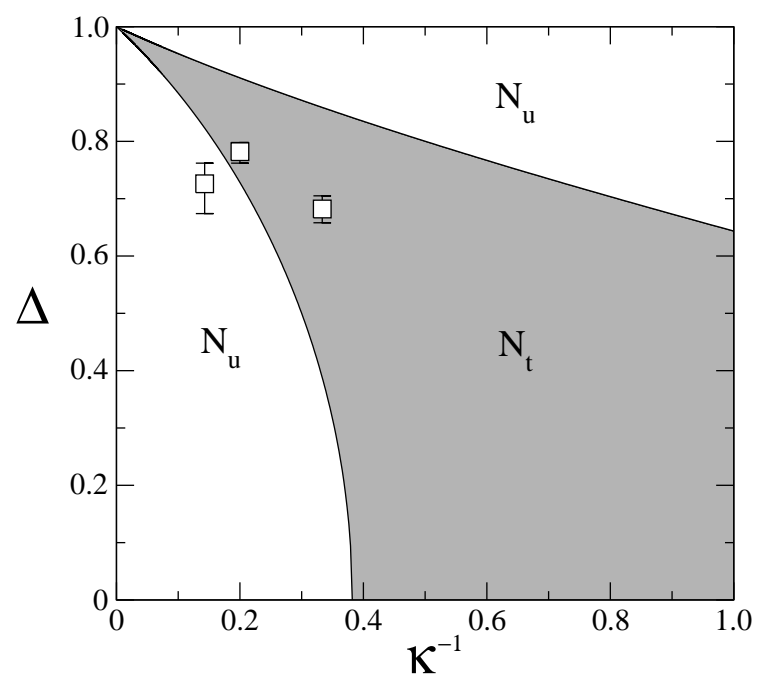

FIG. 5: The functions $\Delta(\kappa)$ in terms of inverse aspect ratio $\kappa^{-1}$. Symbols indicate the nematic phase which is stable in the region in question. The region of tetratic stability is shaded. Open squares indicate values of polydispersity, for values of aspect ratio $\kappa=3,5$ and 7 , at the $\mathrm{I}-\mathrm{N}_{\mathrm{t}}$ transition, as obtained from MC simulation; error bars correspond to uncertainty in $\Delta$ originating from uncertainty in location of spinodal.

the one with minus sign, can be only calculated for $\left.\kappa \geq \kappa^{*}=(3+\sqrt{5}) / 2\right)$. The two lines define a region (shaded in the figure) where the tetratic phase may be stabilised. Thus we see how, as the value of polydispersity $\Delta$ is increased, the maximum value of $\kappa$ for which the tetratic phase ceases to exist increases, which means that polydispersity enhances the formation of tetratic ordering.

Note that the values of $\Delta$ at the upper boundary of the tetratic region are very high, which means that there should be a large number of clusters with their long side $i \sigma_{0} \gg L$, while their width is $L$. This causes the effective average cluster aspect ratio $i \sigma_{0} / L$ to be very high, which induces formation of uniaxial nematic order and thus the $\mathrm{N}_{t}$ phase is destabilised in favour of the $\mathrm{N}_{\mathrm{u}}$ phase. The presence of such big clusters is not observed in the simulations, which means that a more sophisticated model should somehow include additional entropy terms that discourage the formation of big clusters; for the sake of simplicity, we have kept the ingredients of our model to a minimum and avoided any such additional complications.

Finally, in Fig. 5 values of polydispersity $\Delta$ calculated from the simulated cluster size distribution function have been indicated by symbols. These values correspond to the estimated spinodal line of the $\mathrm{I}-\mathrm{N}_{\mathrm{t}}$ transition for the cases $\kappa=3,5$ and 7 . Since these estimations are very rough, the uncertainty in packing fraction at the spinodals goes over to the value of polydispersity (which naturally depends on $\kappa$ and $\eta$ ), which is represented in each case by error bars. We can see that in two of the cases the symbols are well inside the tetratic stability region calculated from the theory. In the case $\kappa=7$, where uncertainty is larger, the MC estimate of $\Delta$ lies outside (but close to) this region.

\section{CONCLUSIONS}

Because of the reduced dimensionality, fluids of twodimensional hard anisotropic particles exhibit strong clustering effects: particles have less freedom to orient in space, which fosters configurations where neighbouring particles lie parallel to each other. However, the impact of this on the onset of new macroscopic symmetries depends very sensitively on the particular geometry of the particles. Thus, in fluids of rectangles, neighbour clusters have a strong tendency to adopt orthogonal relative configurations, since these clusters are almost perfect big rectangles made of several, almost parallel, monomers. These strong tetratic correlations are capable of generating full macroscopic tetratic order and a thermodynamically stable tetratic phase. Therefore, clustering effects are crucial to understand phase behaviour in the HR fluid (and possibly also in the HDR and related fluids), but simple theories at the level of two-body monomer correlations (Onsager, SPT, etc.) cannot account for these effects.

In this work we have presented a simple theory that incorporates clustering in terms of cluster polydispersity, where clusters are considered to be inert particles with no internal degrees of freedom. This assumption may be accurate provided the cluster lifetime (i.e. the average time it takes for a cluster to disappear since it was formed) is longer than the typical cluster diffusion rates in the fluid. Validation of this condition will have to wait for molecular dynamics simulations of the hard-rectangle fluid. Once the fluid is modelled in terms of a multicomponent mixture, one of the available theories for mesophase formation can be used. We have used SPT and have examined the consequence of polydispersity in the phase diagram. As expected, polydispersity enhances the stability of the tetratic phase. Due to the limitations of our model (e.g. the cluster size distribution has to be imposed from outside and does not result from the theory), we cannot make any quantitative comparison with available simulation and experimental results. However, the model can qualitatively explain the formation of tetratic order for rather high values of aspect ratio, as shown by simulation and experiment.

\section{Acknowledgments}

Y.M.-R. gratefully acknowledges financial support from Ministerio de Educación y Ciencia (Spain) under a Ramón y Cajal research contract and the MOSAICO grant. This work has been partly financed by grants Nos. FIS2005-05243-C02-01 and FIS2007-65869-C03-01, also from Ministerio de Educación y Ciencia, and S- 
0505/ESP-0299 from Comunidad Autónoma de Madrid (Spain).

\section{APPENDIX}

In this appendix we provide additional details and further information on the consequences of the model. It contains two sections. In Section A, details on the set of non-linear equations that have to be solved to obtain the equilibrium properties of the HR fluid are provided. Also, the bifurcation analysis of the $\mathrm{I}-\mathrm{N}_{\mathrm{u}, \mathrm{t}}$ transitions is presented. Section B is devoted to discussing the nature of the different phase transitions, together with the behaviour of the distribution functions and to a comparison with simulations. In Section C, a simple chemical model of aggregation is discussed.

\section{A. Minimisation of free energy and bifurcation analysis}

Using Fourier series to represent the orientational distribution functions,

$$
h_{i}(\phi)=\frac{1}{\pi} \sum_{k \geq 0} h_{k}^{(i)} \cos (2 k \phi),
$$

with $h_{0}^{(i)}=1 \forall i$, together with Eqn. (5), we find

$$
S_{i j}=\frac{1}{\pi} \sum_{k \geq 0}\left[\kappa+\frac{i j}{\kappa}+(-1)^{k}(i+j)\right] g_{k} h_{k}^{(i)} h_{k}^{(j)}
$$

where $g_{k}=-\left(1+\delta_{k 0}\right) / 2\left(4 k^{2}-1\right)$. Defining

$$
m_{k}^{(\alpha)}=\sum_{i} x_{i} i^{\alpha} h_{k}^{(i)}, \quad \alpha=0,1,
$$

we obtain

$$
\sum_{i j} x_{i} x_{j} S_{i j}=\frac{\kappa}{\pi} \sum_{k} g_{k} s_{k}^{2}, \quad s_{k}=m_{k}^{(0)}+(-1)^{k} \frac{m_{k}^{(1)}}{\kappa} .
$$

Note that $m_{0}^{(0)}=\sum_{i} x_{i}=1$ while $m_{0}^{(1)}=\sum_{i} x_{i} i$ is the first moment of the discrete cluster size distribution function $\left\{x_{i}\right\}$. Using this notation, the free-energy per particle $\varphi=\Phi / \rho_{0}$ can be written

$$
\begin{aligned}
& \varphi=\ln \left(\frac{y_{0}}{m_{0}^{(1)}}\right)-1+\sum_{i=1}^{n} x_{i}\left\{\ln \left(x_{i} \mathcal{V}_{i}\right)\right. \\
& \left.+\int_{0}^{\pi} d \phi h_{i}(\phi) \ln \left[h_{i}(\phi) \pi\right]\right\}+\frac{y_{0} \kappa}{\pi m_{0}^{(1)}} \sum_{k} g_{k} s_{k}^{2},
\end{aligned}
$$

with $y_{0}=\eta /(1-\eta)$. The functional minimization of (16) with respect to $h_{i}(\phi)$ gives a set of self-consistent non-linear equations which, after some algebraic manipulations, can be transformed into a set of equations for the new variables $s_{k}$ :

$$
\begin{aligned}
& s_{k}=2 \sum_{i=1}^{n} x_{i}\left[1+\frac{(-1)^{k}}{\kappa} i\right] Q_{k}^{(i)}, \\
& Q_{k}^{(i)}=\int_{0}^{\pi} d \phi \cos (2 k \phi) h_{i}(\phi),
\end{aligned}
$$

where the normalized orientational distribution functions are

$$
\begin{aligned}
h_{i}(\phi) & =\frac{e^{-\Lambda_{i}(\phi)}}{\int_{0}^{\pi} d \phi e^{-\Lambda_{i}(\phi)}}, \\
\Lambda_{i}(\phi) & =\frac{4 y_{0} \kappa}{\pi m_{0}^{(1)}} \sum_{k \geq 1} s_{k}\left[1+\frac{(-1)^{k}}{\kappa} i\right] g_{k} \cos (2 k \phi) .
\end{aligned}
$$

The linearization of (17) with respect to $s_{k}(k \geq 1)$ allow us to obtain the expression (6) for the packing fractions at the $\mathrm{I}^{-} \mathrm{N}_{u, t}$ spinodal lines.

\section{B. Phase transitions and distribution functions}

In this subsection we analyse the free energy branches of the model in order to understand the nature of the different phase transitions. The cluster distribution function $x_{i}$ of the mixture is assumed to be exponential, and the width of the distribution is fixed via the polydispersity parameter $q$ (or $\Delta$ ). Eqns. (17)-(20) are solved for different values of $\eta$ to find all metastable and stable phases, either $\mathrm{I}, \mathrm{N}_{\mathrm{u}}$ or $\mathrm{N}_{\mathrm{t}}$ phases. In Fig. 6 the free-energy branches as a function of $\eta^{-1}$ for different values of $q$ (and hence for different polydispersities) are shown. As can be seen, the $\mathrm{N}_{\mathrm{t}}$ phase begins to be stable from $q \approx 0.30$. Also, it is clear that, for $q=0.25$ and 0.35 , the $\mathrm{I}-\mathrm{N}_{\mathrm{u}}$ or $\mathrm{N}_{\mathrm{t}}-\mathrm{N}_{\mathrm{u}}$ transitions are of first order (free-energy branches cross with different slopes). The coexistence values of $\eta$ cannot be determined from the standard double-tangent construction, since the present system is polydisperse. The usual procedure then is to fix the distribution function $x_{i}^{(0)}$ and packing fraction $\eta^{(0)}$ for the parent phase ( $\mathrm{I}$ or $\mathrm{N}_{\mathrm{u}, \mathrm{t}}$ phases) and find the cloud and shadow curves. We have not implemented this procedure here. However, for those values of $q$ for which the transitions are continuous (i.e. $q=0.5$ and 0.65 ), the present procedure adequately determines the transition densities. A similar situation occurs for the cases $\kappa=5$ and $\kappa=7$ (not shown).

It is also interesting to look at the orientational distribution functions of monomers and clusters. From the corresponding functions for clusters of size $i$, i.e. $h_{i}(\phi)$, it is easy to define a cluster orientational distribution 

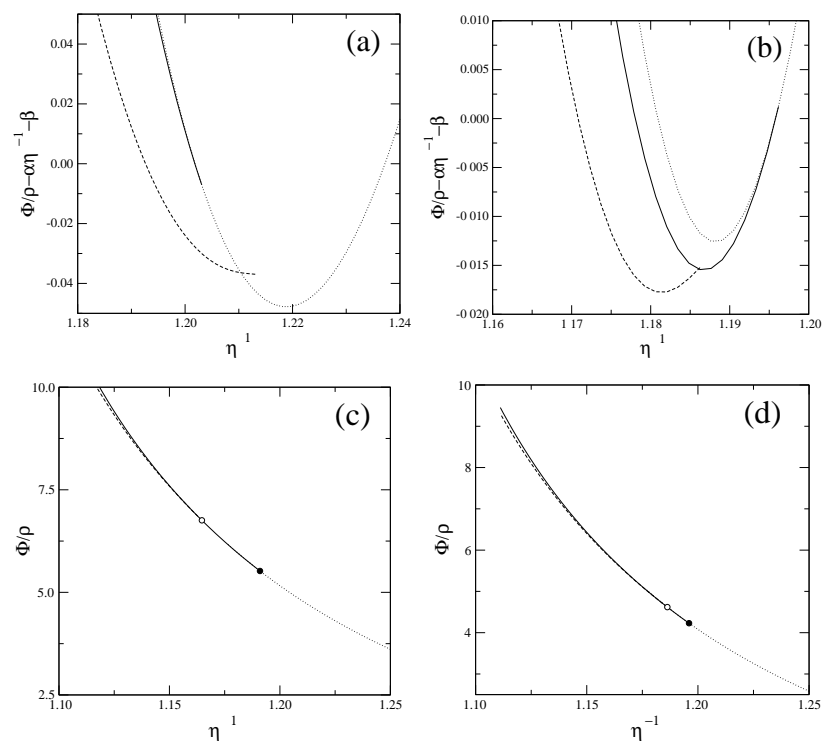

FIG. 6: Free energy per particle $\Phi / \rho$ vs. inverse packing fraction $\eta^{-1}$ from SPT results for the multicomponent HR fluid of monomer aspect ratio $\kappa=3$ and with different values of polydispersity: (a) $q=0.25$, (b) 0.35 , (c) 0.50 and (d) 0.65. Continuous curves: tetratic phase; dashed curves: uniaxial nematic phase; dotted curves: isotropic phase. In (c) and (d) symbols indicate bifurcation points from the isotropic to the tetratic (filled circles) and uniaxial nematic (open circles) phases. In (a) and (b) a straight line in $\eta^{-1}$ has been subtracted to better visualise the curves.

function $h_{c}(\phi)$ as

$$
h_{c}(\phi)=\sum_{i=1}^{n} x_{i} h_{i}(\phi) .
$$

From this, order parameters of the multicomponent mixture can also be defined:

$$
Q^{(k)}=\sum_{i} x_{i} Q_{i}^{(k)}, \quad k=1,2
$$

In the case of monomers the situation is a bit more complicated, since in our model we have lost track of monomers as distinct entities. However, we can simply count the number of monomers pointing along some angle $\phi$ from the set $h_{i}(\phi)$ and then divide by the average number of clusters. Here we have to bear in mind that clusters of size $i$ (having $i$ monomers) with $i<\kappa$ (and $\kappa$ an integer) have a long axis in a direction perpendicular to that of clusters with $i>\kappa$; therefore we write:

$h_{m}(\phi)=\frac{1}{m_{0}^{(1)}}\left[\sum_{i=1}^{n_{0}} x_{i} i h_{i}(\phi)+\sum_{i=n_{0}+1}^{n} x_{i} i h_{i}(\phi+\pi / 2)\right]$,

with $n_{0}=[\kappa]$ (note that in the case where $\kappa$ is not an integer this division has to be done also). Thus the order
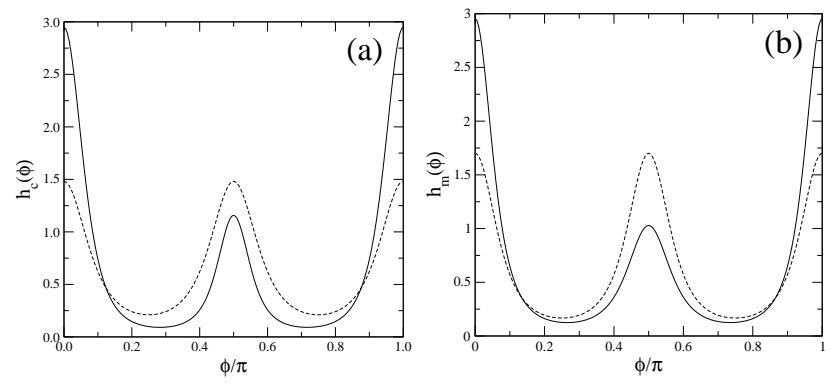

FIG. 7: Cluster (a) and monomer (b) orientational distribution functions for $\kappa=5$ and $q=0.65$. The continuous line corresponds to a stable $\mathrm{N}_{\mathrm{u}}$ phase with $\eta=0.85$, whereas the discontinuous line is for the metastable $\mathrm{N}_{\mathrm{t}}$ phase at the same value of $\eta$.

parameters of the monomers can be calculated as

$$
\begin{aligned}
\langle\cos (2 \phi)\rangle_{m} & =\frac{1}{m_{0}^{(1)}}\left|\sum_{i=1}^{n_{0}} x_{i} i Q_{i}^{(1)}-\sum_{i=n_{0}+1}^{n} x_{i} i Q_{i}^{(1)}\right|, \\
\langle\cos (4 \phi)\rangle_{m} & =\frac{1}{m_{0}^{(1)}} \sum_{i=1}^{n_{0}} x_{i} i Q_{i}^{(2)} .
\end{aligned}
$$

Even for values of aspect ratio for which there exists a region of tetratic stability, the $\mathrm{N}_{\mathrm{u}}$ is always the more stable phase for high values of packing fraction. It is interesting to note that, in this situation, the cluster and monomer distribution functions $h_{\mathrm{c}, \mathrm{m}}(\phi)$ usually have a secondary peak at $\phi=\pi / 2$ corresponding to tetratic ordering (this feature is also present in the simple SPT for the onecomponent HR fluid). Fig. 7 shows these distributions for the case $\kappa=5$ and $q=0.65$. Note that, according to Fig. 5, there exists a stable tetratic phase in this case, with a value of packing fraction at the $\mathrm{N}_{\mathrm{t}}-\mathrm{N}_{\mathrm{u}}$ transition of $\eta^{*}=0.839$. In the figure, the packing fraction chosen is $\eta=0.85>\eta^{*}$ and, therefore, the stable phase has an orientational distribution function pertaining to a $\mathrm{N}_{\mathrm{u}}$. However, both the cluster and the monomer orientational distribution functions of $\mathrm{N}_{\mathrm{u}}$ have secondary peaks. We have also plotted in Fig. 7 the distribution functions of a metastable $\mathrm{N}_{\mathrm{t}}$ at the same value of $\eta$. Finally, it is also interesting that, although the distribution functions $h_{m}(\phi)$ and $h_{c}(\phi)$ in the tetratic region are always tetratic-like (i.e. all maxima have the same height, as it should be by construction), they do not coincide in the uniaxial nematic phase and, consequently, the similarity property at work in the tetratic phase is not obeyed for the uniaxial nematic (see Section II).

\section{Chemical reaction model}

Aggregation phenomena in dilute fluids (e.g. micelle aggregation) are very often described in terms of a chem- 


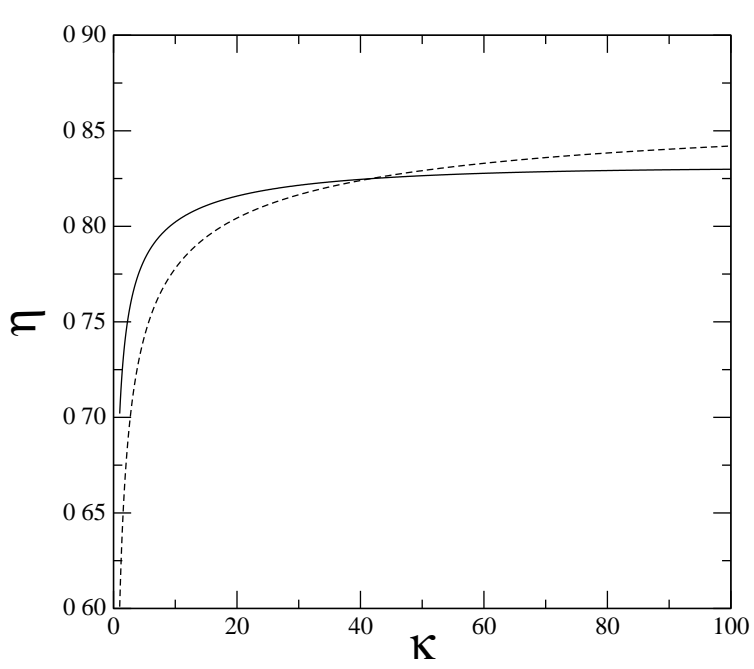

FIG. 8: Packing fractions $\eta$ of the $\mathrm{I}-\mathrm{N}_{\mathrm{t}}$ (continuous line) and $\mathrm{I}-\mathrm{N}_{\mathrm{u}}$ (dashed line) transitions versus aspect ratio $\kappa$.

ical reaction model. An exponentially decaying size distribution immediately emerges from these models. Here we exploit the idea and carry it further using our densityfunctional approximation. This model assumes that the lifetime of a cluster is sufficiently long that it can be defined as a distinct 'chemical' species.

One assumes a chemical reaction of the type $C_{l}+C_{1} \rightleftharpoons$ $C_{l+1}$, with $C_{l}$ denoting a 'chemical' species (i.e. a cluster) containing $l$ monomers. Chemical equilibrium between clusters and monomers then implies the relation

$$
\mu_{i}=i \mu_{1}, \quad i=2, \cdots, n .
$$

Now the chemical potential of the $i$-th species can be calculated from our density-functional theory as

$$
\beta \mu_{i}=\frac{\partial \Phi}{\partial \rho_{i}},
$$

which results in

$$
\begin{aligned}
& \beta \mu_{i}=\ln \left(x_{i} \mathcal{V}_{i}\right)+\int_{0}^{\pi} d \phi h_{i}(\phi) \ln \left[h_{i}(\phi) \pi\right]+\ln y \\
& +y i+2 y \sum_{j} x_{j} S_{i j}+y^{2} S i .
\end{aligned}
$$

The $n-1$ Eqns. (26), together with the condition (2), are a set of $n$ equations with $n$ unknowns $\left(x_{2}, \cdots, x_{n}\right.$ and $\left.\rho\right)$ which allow to find the equilibrium configuration of the fluid. Due to the simplicity of the model an analytical solution can be found.

Since, for the isotropic phase, we have

$$
\sum_{j} x_{j}\left(i S_{1 j}-S_{i j}\right)=\frac{(i-1)}{\pi}\left(\kappa+m_{0}^{(1)}\right),
$$

Eqns. (26), together with (28), give $x_{i}=x_{1} q^{i-1}$ with

$$
q=x_{1} \frac{y_{0}}{m_{0}^{(1)}} \exp \left[\frac{2 y_{0}}{\pi}\left(\frac{\kappa}{m_{0}^{(1)}}+1\right)\right] .
$$

Here we assumed that $\mathcal{V}_{i}=\mathcal{V}_{1}^{i}$ (consistent with the absence of internal degrees of freedom in the clusters and also with the assumption that all clusters have the same mass). Now, since $\sum_{i} x_{i}=1$, we find, for a fluid with an infinite number of species, $1=x_{1} /(1-q)$ which, together with (30), give

$$
x_{1}=\left\{1+\frac{y_{0}}{m_{0}^{(1)}} \exp \left[\frac{2 y_{0}}{\pi}\left(\frac{\kappa}{m_{0}^{(1)}}+1\right)\right]\right\}^{-1} .
$$

Also, the first moment can be calculated self-consistently as

$$
\begin{aligned}
& m_{0}^{(1)}=x_{1} \sum_{i=1}^{\infty} i q^{i-1}=\frac{x_{1}}{(1-q)^{2}} \\
& =1+\frac{y_{0}}{m_{0}^{(1)}} \exp \left[\frac{2 y_{0}}{\pi}\left(\frac{\kappa}{m_{0}^{(1)}}+1\right)\right] .
\end{aligned}
$$

This solution means that $x_{i}=\left(1-1 / m_{0}^{(1)}\right)^{i-1} / m_{0}^{(1)}$. While the first moment $m_{0}^{(1)}$ is the solution of Eqn. (32), the second moment results in

$m_{0}^{(2)}=x_{1} \sum_{i=1}^{\infty} i^{2} q^{i-1}=x_{1} \frac{1+q}{(1-q)^{3}}=m_{0}^{(1)}\left(2 m_{0}^{(1)}-1\right)$.

The polydispersity coefficient, defined as $\Delta=$ $\sqrt{m_{0}^{(2)} /\left(m_{0}^{(1)}\right)^{2}-1}$, turns out to be $\Delta=\sqrt{1-1 / m_{0}^{(1)}}$.

Now the I-N $\mathrm{N}_{u}$ and I-N $\mathrm{N}_{t}$ spinodals can be calculated by solving Eqn. (32), together with the value of $y_{0}$ obtained from Eq. (6). Fig. 8 contains the functions $\eta_{u, t}(\kappa)$ obtained as the solutions of (32) and (6). As can be seen the uniaxial nematic is more stable than the tetratic up to $\kappa^{*} \approx 41.65$. This results from the peculiar behaviour of the area distribution function $i x_{i}$ : its zeroth-order moment is always greater than $\kappa$, while it is larger in the tetratic phase when $\kappa<\kappa^{*}$ [Fig. 9(a)]; this behaviour is inverted for $\kappa>\kappa^{*}$, and the moment becomes larger for the uniaxial nematic phase, as can be seen in Fig. 9(b). Again this peculiar behaviour is due to the relatively high proportion of very big clusters, with $i \sigma_{0}>L$, which stabilise the $\mathrm{N}_{u}$ phase against the $\mathrm{N}_{t}$ phase. This behaviour is not observed in simulations, because the formation of very big clusters is penalised by fluctuations. The model has the value that an exponential cluster distribution function is predicted. 

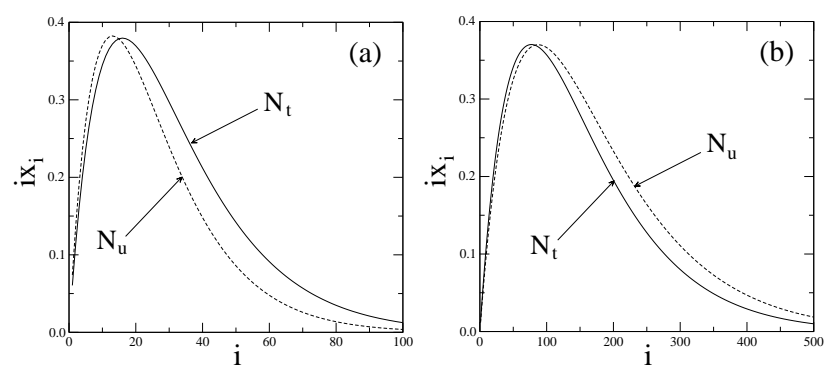

FIG. 9: Area distribution functions for tetratic $\mathrm{N}_{\mathrm{t}}$ and uniaxial $\mathrm{N}_{\mathrm{u}}$ nematic phases. (a) $\kappa=10$ and (b) $\kappa=100$.

[1] K. Zhao, C. Harrison, D. Huse, W. B. Russel, and P. M. Chaikin, Phys. Rev. E 76, 040401(R) (2007).

[2] H. Schlacken, H.-J. Mogel, and P. Schiller, Mol. Phys. 93, 777 (1998).

[3] Y. Martínez-Ratón, E. Velasco, and L. Mederos, J. Chem. Phys. 122, 064903 (2005).

[4] K. W. Wojciechowski and D. Frenkel, Comp. Mat. Sci. Tech. 10, 235 (2004).

[5] A. Donev, J. Burton, F. H. Stillinger, and S. Torquato, Phys. Rev. B 73, 054109 (2006).
[6] D. A. Triplett and K. A. Fichthorn, Phys. Rev. E 77, 011707 (2008).

[7] V. Narayan, N. Menon, and S. Ramaswamy, J. Stat. Mech. P01005 (2006).

[8] Y. Martínez-Ratón, E. Velasco, and L. Mederos, J. Chem. Phys. 125, 014501 (2006).

[9] M. A. Bates and D. Frenkel, J. Chem. Phys. 112, 10034 (2000). 